\title{
Active coated nano rod antennas for enhanced and directive scattering phenomena
}

\author{
Samel Arslanagić, ${ }^{1, *}$ and Rasmus E. Jacobsen ${ }^{2}$ \\ ${ }^{1}$ Department of Electrical Engineering, Technical University of Denmark, Kgs. Lyngby 2800, Denmark \\ 2 Department of Photonics Engineering, Technical University of Denmark, Kgs. Lyngby, 2800, Denmark
}

Received: 8 February 2019 / Accepted: 9 September 2019

\begin{abstract}
The scattering properties of a range of symmetric and asymmetric active coated nano rod antennas are investigated numerically. The active nano rods are composed of a silica dioxide nano-core coated with a silver nano-shell, and with a canonical gain model implemented into their nano-core regions. The asymmetric nano rods are obtained through suitable perforations of their nano-shell and/or nano-core regions. In all cases, active nano rods are found to exhibit super-resonant phenomena with significantly enhanced scattered fields for an incident plane wave having the magnetic field parallel to the rod axis. While the dipole-mode response in the symmetric cases is only weakly directive, the asymmetric cases stimulate an abundant emission of higher order modes furnishing rather enhanced and directive near-fields. As the length of the symmetric nano rods decreases, more gain is needed to achieve a super-resonant response, which also was found to be blue-shifted. For asymmetric cases, the gain was lowered, and the response got blue-shifted as the asymmetry increased. The proposed active nano rod antennas provide a new class of antennas with desirable wavelength tunability and polarization-dependent scattering properties; this makes them interesting candidates for many nano-photonic applications. Moreover, the proposed geometries bridge the important gap between the two often considered canonical geometries, namely, spherical and infinitely long cylindrical particles. The detailed knowledge of gain values and resonant wavelengths provided in here is crucial for a successful combination of such particles with realistic gain materials.
\end{abstract}

Keywords: Symmetric resonators / asymmetric resonators / gain / nano rods / nano antennas / scattering

\section{Introduction}

Our ability to tailor interactions between light and matter on the nano-scale may have a profound impact on a variety of nano-photonic fields, such as nano-sensors and lasers, absorbers and solar cells, artificial materials, optical magnetism, cloaking, optical communication, imaging and spectroscopy, biology and medicine, to mention a few [1-13]. Nano-antennas (NAs) are the key enabling technology for effective light-matter interactions at the nano-scale. They enable efficient conversion of incident light into a strongly localized/confined field, as well as the efficient conversion of the emissions from weak sources into intense, directed propagating waves.

Apart from the realizations of NAs in terms of scaleddown versions of their microwave counterparts [14-16], notable attention was devoted to NAs based on plasmonic and/or all-dielectric high-index particles [15-22]. Although high-index all-dielectric particles give rise to efficient and

\footnotetext{
* e-mail: sar@elektro.dtu.dk
}

low-loss designs of optical NAs, the plasmonic counterparts lead to NAs with rather profound field enhancements and localizations even in deeply sub-wavelength structures. The latter is due to the occurrence of surface plasmons at plasmonic interfaces [23]. However, the full potential and performance of plasmonic-based NAs is to some extent hindered by the large plasmonic losses at optical frequencies. To circumvent the loss issue, the use of gain materials was proposed in a variety of active plasmonic particles [24-26] for which significantly enhanced radiation and scattering characteristics were reported.

A large number of initial particle-based NAs involved sub-wavelength sizes with the resulting fundamental dipole mode of operation which is only weakly directive. However, many applications are in high demand of devices that enable efficient flow of energy in preferred directions, justifying the need for directive NAs. In this regard, both symmetric and asymmetric configurations have been considered for a variety of the above mentioned material choices. In the symmetric cases, either core-shell or multilayered particles were utilized to excite simultaneously electric and magnetic dipole modes (to realize a Huygen's 
source) or to excite even higher order modes, thereby boosting the directivity of such particles [27-30]. On the other hand, introducing asymmetry, by either off-setting the material layers $[31,32]$ or by introducing appropriate perforations of the particles [33,34], was also found to stimulate the excitation of higher order modes, and thus to lead to a more interesting as well as directional performance characteristics. In addition hereto, super-directive behavior with needle-like radiation characteristics was recently demonstrated theoretically in spherical [35] and cylindrical [36] multi-layered structures.

As to the active plasmonic-based NAs, most attention has thus far been devoted to three-dimensional (3D) spherical and two-dimensional (2D) circularly cylindrical active coated nano particles (CNPs). Owing to their enhanced responses, they were termed super-resonant particles and were said to possess the so-called superresonance. While the 3D spherical CNPs are realistic in terms of their shape, their properties are not polarization dependent. This limits their potential for a number of applications. On the other hand, the 2D cylindrical CNPs are strongly polarization sensitive, but are not realistic due to their infinite extent along one of the dimensions.

The purpose of the present work is twofold. We first account for the super-resonant properties in 3D truncated circularly cylindrical active CNPs with symmetric material arrangements. These are presently referred to as symmetric active coated nano rods (CNRs). To provide fundamental insight into these new NA geometries, symmetric CNRs of three different lengths will be examined numerically and compared with the canonical 2D cylindrical and 3D spherical CNPs. By doing so, we also bridge the gap between these two often considered canonical problems as the proposed CNRs lie in between the two on the account of their geometry. We then introduce suitable perforations in the nano-shell and then also in the nano-core to form asymmetric active CNRs and examine numerically their potential in providing, not only enhanced, but also directive scattering characteristics. In all cases, the nano-core will be made of a silica-dioxide dielectric material, while the nano-shell will be made of silver. Furthermore, a constant frequency gain model will be used in the nano-core region of the CNRs. Both symmetric and asymmetric CNRs will be shown to be super-resonant with rather large values of the total scattering cross section when a plane wave having the magnetic field parallel to the rod axis is incident on them. In the symmetric cases, the response is due to a resonant dipole mode, while the asymmetric cases will be shown to lead to rather directive radiation properties in the vicinity of the CNR antenna due to the excitation of higher order modes. Shorter symmetric CNRs are found to require more gain than the longer ones, and to have a response which is blue-shifted. In the asymmetric cases, the amount of gain diminishes and the response gets blue-shifted with an increased asymmetry of the CNR.

Related symmetric CNR of a fixed dimension with gold in its nano-core was examined previously in [37]. Symmetric CNRs of the same material composition but somewhat different geometrical shape have been investigated in $[38,39]$ for a fixed length of the particle. In contrast to these works, our systematic approach provides, however, a detailed knowledge on the required gain values as well as the resonant wavelengths as we transition gradually from 2D cylindrical, over 3D CNR, to 3D spherical active designs. This may prove indispensable in successful realistic gain media implementation in the proposed CNRs. By simply adjusting the length of the CNRs, their super-resonances can be tuned to overlap spectrally with the emission lines of possible gain materials, thereby attaining realistic super-resonant CNR peak responses in line with the results presented in here. In addition to this fundamental insight, the proposed active CNRs provide a new class of antennas with not only desirable wavelength tunability and polarization-dependent properties but also with highly directional responses. This makes them interesting candidates for a variety of nano-photonic applications, e.g., optical wireless systems, nano-sensors, and single photon sources, to mention a few.

The present manuscript is organized as follows. Section 2 introduces the CNR configurations, together with the used material and gain models. This section also discusses the established numerical model used to perform the analysis, and introduces the quantities of interest used to characterize the examined CNRs. Section 3 includes a discussion of the results for symmetric and asymmetric CNRs. Finally, the work is summarized and concluded in Section 4. Throughout the manuscript, the time factor $\exp (j \omega t)$, with $\omega$ being the angular frequency and $t$ being the time, is assumed and suppressed.

\section{The coated nano rod problem and its solution}

\subsection{Configuration, materials and gain model}

The coated nano rod (CNR) configuration of interest to this work is shown in Figure 1. It consists of a nano-core (yellow, region 1) which is covered by a nano-shell (red, region 2). Two variations of the CNR will be considered, namely, the symmetric and the asymmetric one. The symmetric CNR consists of a circular cylindrical core-shell part of length $h$; the nano-core radius of this part is denoted by $a$ while the outer radius of its nano-shell is denoted by $b$, see Figure 1a and b. The cylindrical core-shell part is completed, on the top and bottom, with a hemi-spherical core- shell part of the same material composition and coreshell dimensions as the cylindrical core-shell part. The symmetric CNR will be contrasted to the asymmetric one; the cross-sectional views of their cylindrical core-shell parts are shown in Figure 1c and d. These are the so-called holey and cake CNRs, respectively. They follow from the symmetric CNRs through angular sector perforations of their nano-shell and then also of the nano-core by an amount specified by the cut-out angle $\theta$. The material composition as well as the over-all size of the asymmetric CNRs (as specified by the height $h$ and the two radii $a$ and $b$ ) remain unchanged relative to the symmetric case.

The CNRs are embedded in free-space (region 3) with permittivity $\varepsilon_{0}$, permeability $\mu_{0}$, and thus the wave number $k_{0}=\omega \sqrt{\varepsilon_{0} \mu_{0}}=2 \pi / \lambda$, with $\lambda$ denoting the free-space 


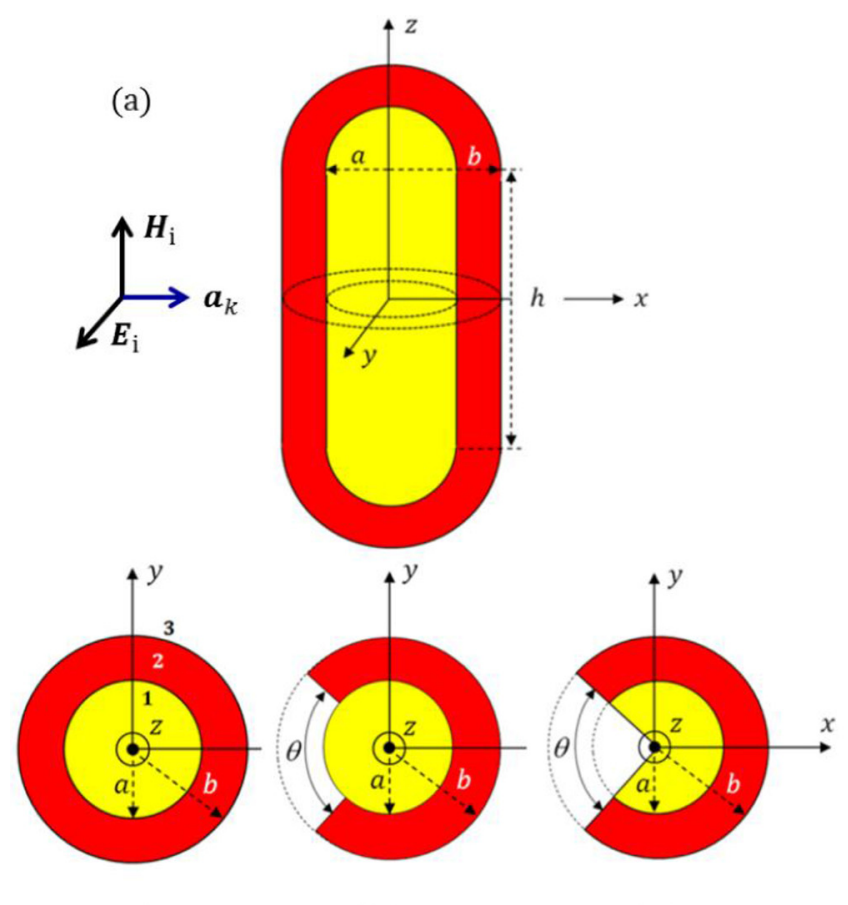

(b) (c) (d)

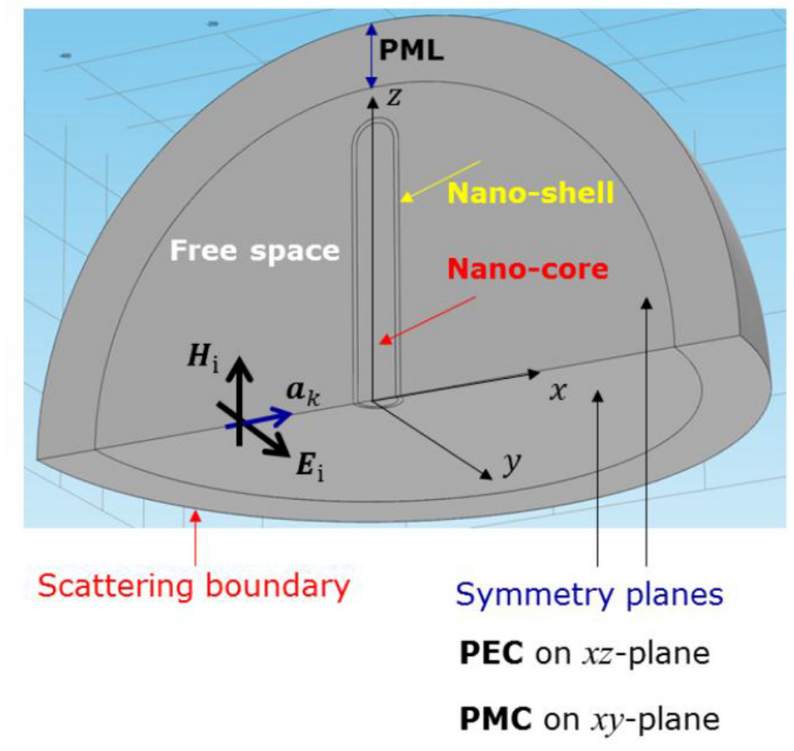

(e)

Fig. 1. The examined CNR configurations (a)-(d), and the numerical model of the plane wave excited symmetric CNR (e).

wavelength. The nano-core region of all CNRs is made of silica-dioxide $\left(\mathrm{SiO}_{2}\right)$. This dielectric material has the permittivity $\varepsilon_{n c}=2.05 \varepsilon_{0}$ and permeability $\mu_{n c}=\mu_{0}$. The nano-shells are plasmonic and are all made of silver $(\mathrm{Ag})$ with the complex permittivity $\varepsilon_{n s}=\varepsilon_{n s}^{\prime}-j \varepsilon_{n s}^{\prime \prime}$ and permeability $\mu_{n s}=\mu_{0}$. In all numerical investigations focus is placed on Ag nano-shells of $6 \mathrm{~nm}$ thickness. Such a small nanometer thickness requires, however, very accurate sizedependent permittivity models of the plasmonic nanoshells. Appropriate permittivity models have been developed and used successfully for a range of nano-shell thicknesses; the specific behavior of the permittivity pertaining to a $6 \mathrm{~nm}$ thick $\mathrm{Ag}$ nano-shell used in this work can be found in e.g., $[24,26]$. Over the visible range of wavelengths, $450-700 \mathrm{~nm}$, which is sufficient for the illustration of all results for the light-CNR interactions examined presently, the real part of the Ag permittivity is negative while its imaginary part implies a rather lossy behavior.

The rather lossy feature of the Ag nano-shell has inevitable effects of the potential resonant properties of the CNRs. In order to enhance their radiation and scattering characteristics, the plasmonic losses must be overcome. To this end, the nano-core of the CNRs will be impregnated with a gain material. More specifically, use will be made of a constant frequency gain model. According to this model, the nano-core permittivity is given by $\varepsilon_{n c}=\varepsilon_{0}\left(n^{2}-\kappa^{2}-\right.$ 2jnк) where $n=\sqrt{2.05}$ is the refractive index of the $\mathrm{SiO}_{2}$ nano-core maintained at its value for the lossless nano-core case. The parameter $\kappa$ represents the loss/gain parameter/ coefficient. It thus determines the nature of the CNRs; when $\kappa=0$ and $\kappa>0$ the CNRs are passive, while for $\kappa<0$ the CNRs are active. In our study, the attention is devoted only to active CNRs due to the inefficiency of the passive cases to achieve enhanced resonant phenomena in such configurations.

The constant frequency gain model is chosen presently with the aim of obtaining the maximum response of the investigated active CNRs. The values of the gain parameter, $\kappa$, reported in here represent achievable gains by e.g., commercially available quantum dots [40]. It is known that realistic gain materials are, however, dispersive. Their presence will, moreover, detune the constant gain response characteristics of the CNRs. For an optimal response in a practical setup, the resonances of the CNRs and the gain media must therefore spectrally overlap as much as possible; this can be facilitated through challenging fine tuning of the size of the examined CNRs impregnated with realistic gain materials.

\subsection{Solution and quantities of interest}

The CNRs are investigated under their excitation with a uniform plane wave as indicated in Figure 1a. To connect the CNR geometries to the features of the plane wave excitation, a rectangular $(x, y, z)$ coordinate system has been introduced with its origin coinciding with the center of the CNR, and its z-axis being directed along CNR axis. The polarization of the plane wave is transverse electric (TE) with the incident electric and magnetic fields given by

$$
\begin{gathered}
\boldsymbol{E}_{i}=\boldsymbol{a}_{y} E_{0} e^{-j k_{0} x}, \\
\boldsymbol{H}_{i}=\boldsymbol{a}_{z}\left(E_{0} / \eta_{0}\right) e^{-j k_{0} x},
\end{gathered}
$$


respectively. The quantity $E_{0}[\mathrm{~V} / \mathrm{m}]$ denotes the magnitude of the incident electric field while $\eta_{0}$ is the intrinsic impedance of free space given by $\eta_{0}=\sqrt{\mu_{0} / \varepsilon_{0}}$. The CNR gives rise to fields scattered in all three regions. The total fields, which are the sum of the incident and scattered fields, are denoted by $\boldsymbol{E}$ for the electric field, and $\boldsymbol{H}$ for the magnetic field; in general, they possess all three $(x, y$, and $z)$ field components.

The problem at hand is solved numerically using the full-wave Comsol Multiphysics modeling software [41], which is based on the finite-element method. To this end, a three-dimensional design domain is utilized; its graphical visualization is shown in Figure 1e for the plane wave excited symmetric CNR. As a matter of fact, the figure shows only a quarter of the entire configuration since symmetry planes (perfect electric conductor in the $x z$-plane and perfect magnetic conductor in the $x y$-plane) are used to reduce the computational load. A similar numerical model is established for the holey and cake asymmetric CNR. However, in those cases symmetry exists only with respect to the $x y$-plane. As observed in Figure 1e, the CNRs are located in a free space domain that is terminated with a shell of a perfectly matched layer (PML). The inner surface of the PML is the integration surface used to obtain a number of derived quantities to be described below. The outer surface of the PML shell is replaced with a scattering boundary to further suppress any fields reflected from it. The distance to the PML was set to $120 \mathrm{~nm}$ in the $x y$-plane while it was varied in the $x z$-plane depending on the CNR height. The PML shell thickness $(70 \mathrm{~nm})$ as well as the mesh-fineness (in particular on the integration surface and within the CNR) was in all cases selected such as to ensure the convergence and accuracy of the final solution.

Once the unknown fields in all regions have been determined, they can be used, together with a set of derived quantities to quantify the performance of the CNRs. To complement the near-field analysis, the total power flow density given by

$$
\boldsymbol{S}(x, y, z)=(1 / 2) \operatorname{Re}\left\{\boldsymbol{E} \times \boldsymbol{H}^{*}\right\},
$$

will also be used. In addition to the near-field analysis, the far-field properties of the CNRs will also be addressed. To this end, we will use the total scattering cross section $(\mathrm{SCS}), \sigma_{t}$, given by

$$
\sigma_{t}=P_{s} / S_{i}
$$

where $P_{s}$ is the scattered power and $S_{i}=\left|E_{0}\right|^{2} / 2 \eta_{0}$ is the incident power flow density. The scattered power $P_{s}$ is obtained as the flux of the scattered power flow density through a surface (e.g., integration surface) enclosing the CNR. In our numerical investigations, the total SCS will be stated in $\mathrm{dB}$ as follows

$$
\sigma_{t}=10 \log _{10}\left(\sigma_{t} / A\right) \quad[\mathrm{dBcs}](3 \mathrm{D} \mathrm{CNP}),
$$

where $\mathrm{dB}$ cs stands for the decibels relative to the crosssectional area of the CNR, $A=\pi b^{2}$. As to the asymmetric CNRs, their directional response will furthermore be quantified by the scattering cross section in the maximum direction. To this end, the differential scattering cross section given by

$$
\sigma_{d}(\theta, \phi)=\lim _{r \rightarrow \infty} 4 \pi r^{2}\left|\boldsymbol{H}_{s}\right|^{2} /\left|\boldsymbol{H}_{i}\right|^{2},
$$

is introduced, with $r$ being the radial coordinate of the spherical $(r, \theta, \phi)$ coordinate system (associated in the usual manner to the rectangular $(x, y, z)$ coordinate system), while $\boldsymbol{H}_{s}$ is the field scattered from the CNRs. Stated in $\mathrm{dB}$, the scattering cross section in the maximum direction reads

$$
\sigma_{d, \max }=\max _{\theta, \phi} 10 \log _{10}\left(\sigma_{d} / A\right)[\mathrm{dBcs}] .
$$

Before we proceed with the results pertaining to a variety of CNRs, following remarks are in order. First, when the length of the cylindrical core-shell part of the symmetric CNR is $h=\infty$, see Figure 1a, then the symmetric CNR goes over to a $2 \mathrm{D}$ cylindrical coated nano particle (CNP) investigated analytically in [26]. On the other hand, when $h=0$, the symmetric CNR recovers a 3D spherical CNP studied by analytical means in $[24,26]$. The results for these two canonical problems will inhere be contrasted to those obtained for various CNRs. This will further enhance our understanding of the underlying light-matter interactions as the gap between the two often considered canonical problems will be bridged by the proposed CNRs which lie in between on the account of their geometry. In addition, the analytical results will provide appropriate reference cases which are used to validate the established numerical models.

\section{Results and discussion}

As already mentioned, efforts have thus far been devoted to a detailed analysis of symmetric $2 \mathrm{D}$ cylindrical and $3 \mathrm{D}$ spherical CNPs (obtained for $h=\infty$ and $h=0$, respectively, in Fig. 1a and b) utilizing not only silver, but also gold and copper as the plasmonic material $[24,26]$. By including gain, and thus making them active, significantly enhanced radiation and scattering characteristics were reported for such particles despite of their electrically small crosssectional sizes. The enhanced response was primarily due to the excitation of a strong resonant dipole mode in their interior, and the active particles were said to be superresonant. Attention was devoted mostly to particles having silica-dioxide in their nano-cores and silver in their nanoshell, and with the dimensions selected as $-a=24 \mathrm{~nm}$ (nano-core radius),

$-b=30 \mathrm{~nm}$ (overall coated nano particle radius).

The super-resonant behavior for the symmetric $2 \mathrm{D}$ cylindrical and 3D spherical active Ag-based CNPs was found for specific values of the gain parameter, $\kappa$, and the resonant wavelength, $\lambda$, summarized in Table 1 .

Our aim in what follows is to first describe the outcomes of the corresponding active Ag-based CNRs which are the finite, 3D counterparts of the above mentioned symmetric 2D Ag-based cylindrical CNP, and then to account on the effects of asymmetry as introduced in the holey and cake 


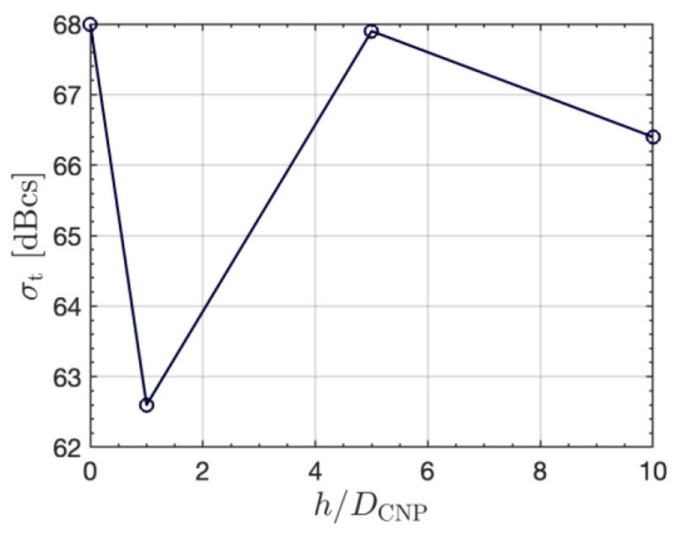

(a)

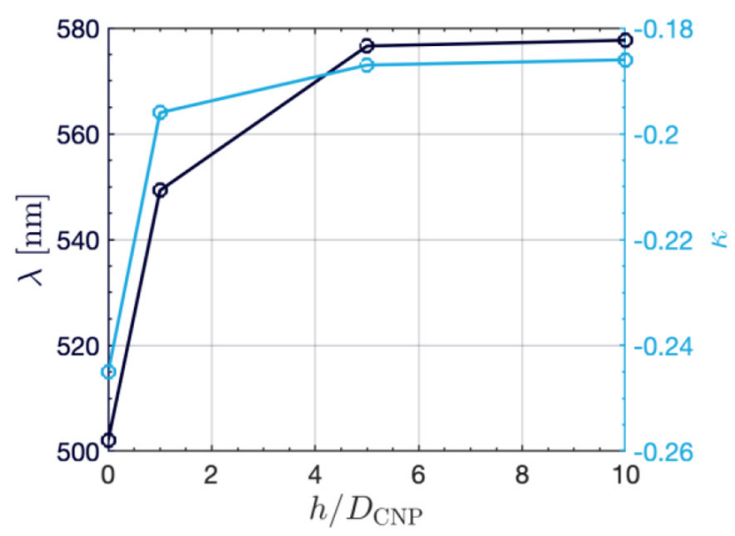

(b)

Fig. 2. The total scattering cross section, $\sigma_{t}$, as a function of the length $h$ of the cylindrical core-shell part for the super-resonant, symmetric and active Ag-based 3D CNRs (a). The corresponding resonant wavelength, $\lambda$, and the gain parameter, $\kappa$, are shown in (b). The circles indicate calculated values.

Table 1. The gain parameter, $\kappa$, and the resonant wavelength, $\lambda$, for super-resonant $2 \mathrm{D}$ circularly cylindrical and 3D spherical CNPs $[19,21]$.

\begin{tabular}{lll}
\hline CNP & $\kappa$ & $\lambda[\mathrm{nm}]$ \\
\hline 2D & -0.175 & 577.7 \\
3D & -0.245 & 502.1 \\
\hline
\end{tabular}

CNRs. Since the CNRs are obtained through an appropriate truncation of the 2D case, their material composition (as was also described in Sect. 2.1) and their cross-sectional size in the $x y$-plane remain unchanged, i.e., $a=24 \mathrm{~nm}$ and $b=30 \mathrm{~nm}$, see also Figure 1a-d.

\subsection{Symmetric CNR antennas}

Three different sizes of the symmetric CNRs will be considered. The length $h$ of the cylindrical core-shell part was chosen as,

$$
h=\left\{1 D_{\mathrm{CNR}}, 5 D_{\mathrm{CNR}}, 10 D_{\mathrm{CNR}}\right\}
$$

where $D_{\mathrm{CNR}}=2 b=60 \mathrm{~nm}$ is the diameter of the CNR in the $x y$-plane, see Figure 1a and b. Since the cylindrical coreshell part is terminated at both ends with a core-shell hemisphere of the same dimensions, the overall CNR lengths are $H=\left\{2 D_{\mathrm{CNR}}, 6 D_{\mathrm{CNR}}, 11 D_{\mathrm{CNR}}\right\}$. All CNRs are illuminated by a TE polarized plane wave in (1) with $E_{0}=1[\mathrm{~V} / \mathrm{m}]$.

Our extensive numerical studies and optimizations have shown that symmetric active CNRs can be superresonant and lead to significantly enhanced scattering characteristics as the losses become overcome by the gain inclusion inside their nano-cores. This is evidenced by the very large total scattering cross section, $\sigma_{t}$, from (3) depicted in Figure 2a as a function of $h$ values for the examined 3D CNRs. The corresponding results for the gain parameter, $\kappa$, and the wavelength, $\lambda$, at which
Table 2. The total scattering cross section, $\sigma_{t}$, the gain parameter, $\kappa$, and the resonant wavelength, $\lambda$, of the superresonant symmetric active Ag-based CNRs.

\begin{tabular}{llll}
\hline$h[\mathrm{~nm}]$ & $\sigma_{t}[\mathrm{dBcs}]$ & $\kappa$ & $\lambda[\mathrm{nm}]$ \\
\hline$\infty$ & $47.7[\mathrm{dBc}]$ & -0.175 & 577.7 \\
$10 D_{\mathrm{CNR}}$ & 66.4 & -0.186 & 577.7 \\
$5 D_{\mathrm{CNR}}$ & 67.9 & -0.187 & 576.6 \\
$1 D_{\mathrm{CNR}}$ & 62.6 & -0.196 & 549.3 \\
0 & 68.0 & -0.245 & 502.1 \\
\hline
\end{tabular}

the enhanced cross section values are attained, are shown in Figure 2b.

For easier reference, Table 2 presents a summary of all relevant values for these symmetric active Ag-based CNRs. For reference purposes, the corresponding results for the 2D cylindrical and 3D spherical active CNPs are also included in the table. These reference values have presently been obtained numerically, and are found to fully agree with the previously reported analytical results $[24,26]$, thereby confirming the validity of the established numerical model. In the 2D case, the total scattering cross section, also known as the scattering width, has also been determined by (3). However, rather than normalizing to the cross-sectional area $A$ as in (4) prior taking the logarithm, it was normalized to the circumference $C=2 \pi b$ of the $2 \mathrm{D}$ cylindrical coated nano particle. The decibel scale in this case is thus indicated by $[\mathrm{dBc}]$.

As observed in Table 2, very large and similar values of $\sigma_{t}$ are obtained for all CNRs. The notable enhancements in the active cases are further emphasized by noting that e.g., a symmetric passive CNR with $h=5 D_{\mathrm{CNR}}$ exhibits a total cross section of about $\sigma_{t}=10.3 \mathrm{dBcs}$. This is more than $50 \mathrm{dBcs}$ smaller than the corresponding active case. We also find that the amount of gain required for the superresonant behavior increases with the decreasing particle length $h$ due to their smaller physical volume of the gain 


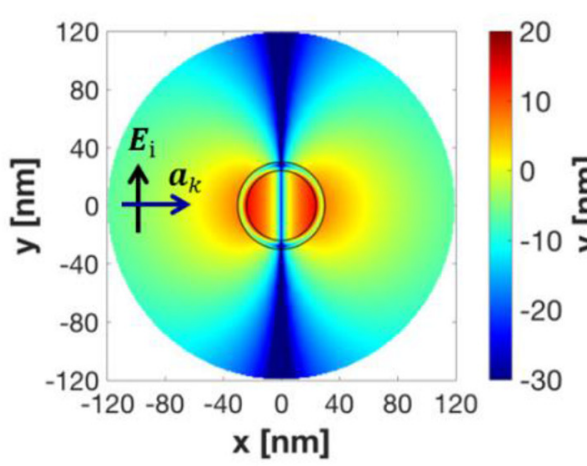

(a)

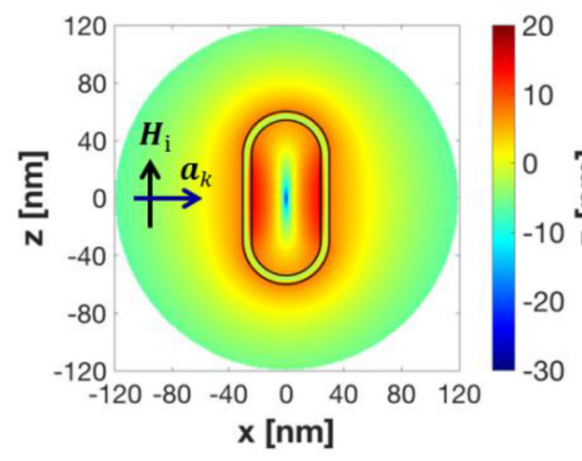

(d)

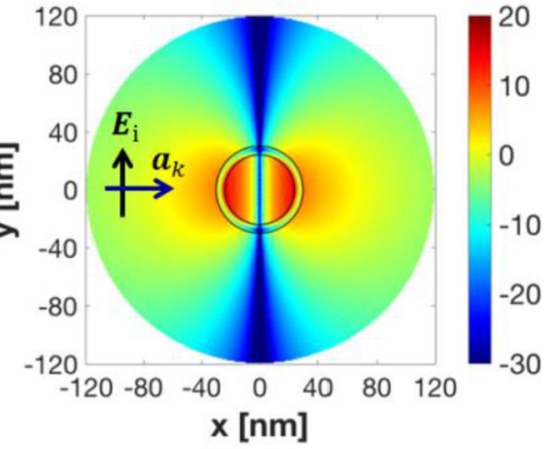

(b)

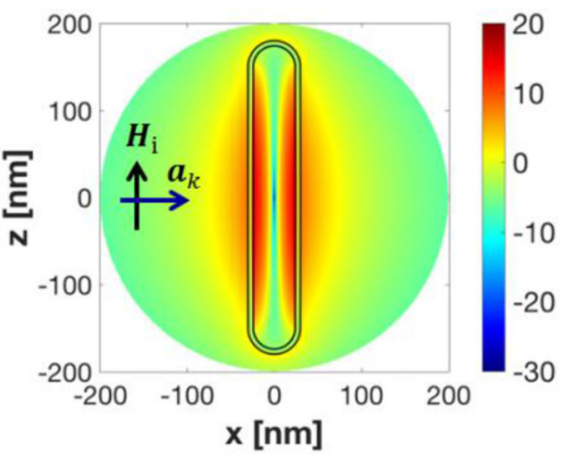

(e)

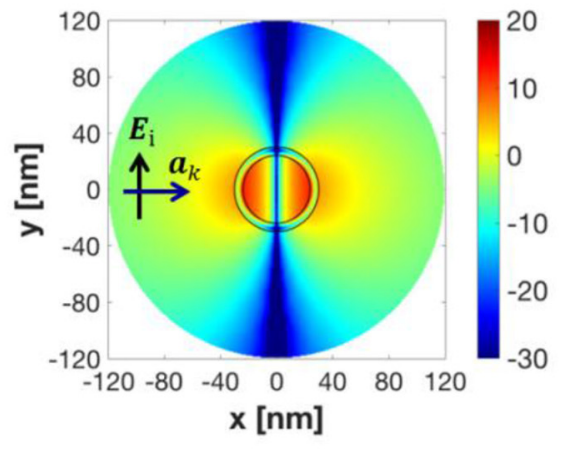

(c)

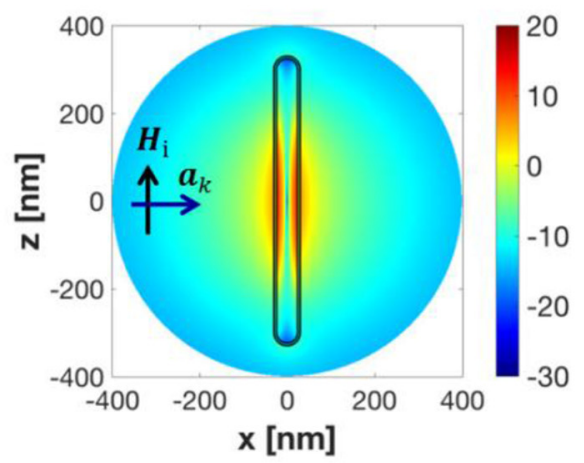

(f)

Fig. 3. The magnetic field, $20 \log |\boldsymbol{H} / 1[\mathrm{~A} / \mathrm{m}]|$, of the symmetric super-resonant Ag-based CNRs for $h=1 D_{\mathrm{CNR}}$, (a) and (d); $h=5 D_{\mathrm{CNR}}$, (b) and (e); and $h=10 D_{\mathrm{CNR}}$, (c) and (f). The results are shown in the $x y$-plane (upper row) and the $x z$-plane (lower row). Curves representing the surfaces of the CNRs are included in each figure. The insets depict the direction of the incident fields and wave propagation $\left(\boldsymbol{a}_{k}=\boldsymbol{a}_{x}\right)$.

region. Moreover, the super-resonant wavelength is blueshifted as the particle length gets decreased. In slightly different words, the longer active CNRs attain their superresonant behavior at longer wavelengths and require less gain in contrast to shorter CNRs. This knowledge is useful for the purpose of successful realistic gain implementation in these particles, e.g., by using quantum-dot materials. By adjusting the height of the CNRs, their super-resonances can be tuned to overlap spectrally with the emission lines of quantum-dot materials, thereby attaining realistic superresonant CNR peak responses in accordance with the results presented in here.

To unveil the origin of the super-resonant behavior of the active CNRs, Figure 3 shows the associated magnetic field, $20 \log |\boldsymbol{H} / 1[\mathrm{~A} / \mathrm{m}]|)$, for the three sizes $h=1 D_{\mathrm{CNR}}$, $5 D_{\mathrm{CNR}}$, and $10 D_{\mathrm{CNR}}$. The results pertaining to the $x y$-plane of the CNRs are shown in the upper row, while those in the $x z$-plane are shown in the lower row.

The field responses in the $x y$-plane all display a strong dipole mode. The field results in the $x z$-plane also show a strong response, this being particularly so near the surfaces of the nano-shell. For the shortest CNR, $h=1 D_{\mathrm{CNR}}$, the field is rather large and homogeneous along the entire part of the nano-shell surfaces. However, there is a notable field decrease away from the particle center for longer CNRs. This is mostly profound for the longest, $h=10 D_{\mathrm{CNR}}$, CNR case. The total length of the longest CNR is $660 \mathrm{~nm}$, which is somewhat larger than its resonant wavelength of $577.7 \mathrm{~nm}$. These results show that the ends of long CNRs are not effectively exploited in the scattering process. As such, they may indicate optimal placement of possible quantum-dot materials in realistic CNR configurations.

We note that the super-resonant response is obtained for an incident plane wave having the magnetic field parallel to the nano rod axis. Upon the change of polarization, with the electric field being parallel to the nano rod axis, very weak response was in evidence. Thus, even the shortest, $=1 D_{\mathrm{CNR}}$, CNR, which is not far from the spherical shape is strongly polarization dependent due to the presence of gain. Thus, the proposed active CNRs are strongly polarization-dependent, making them useful for e.g., sensor applications.

\subsection{Asymmetric CNR antennas}

The results of the previous section have clearly demonstrated that symmetric active CNRs can be designed to be super-resonant with significantly enhanced total scattering cross section. However, since they arise from their fundamental dipole mode excited in their interiors, such CNRs are not very directive. To this end, the proposed 


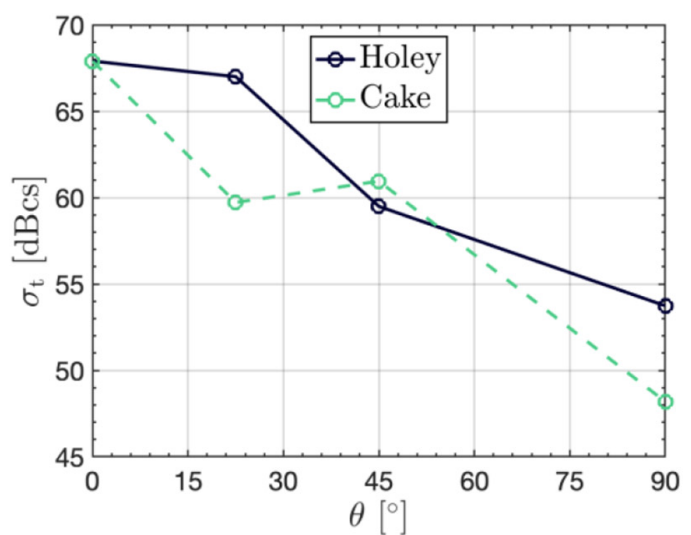

(a)

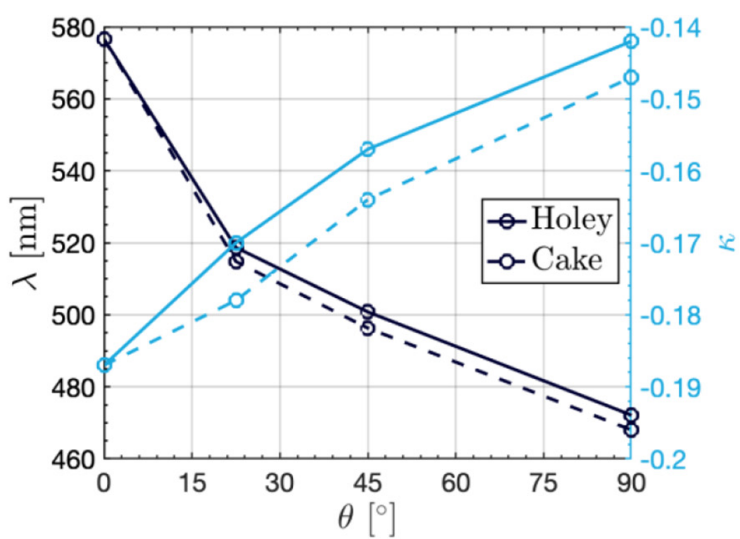

(b)

Fig. 4. The total scattering cross section, $\sigma_{t}$, as a function of the cut-out angle, $\theta$, for the super-resonant, asymmetric holey and cake active Ag-based CNRs (a). The corresponding resonant wavelength, $\lambda$, and the gain parameter, $\kappa$, are shown in (b). The circles indicate calculated values.

Table 3. The total scattering cross section, $\sigma_{t}$, the scattering cross section in the maximum direction, $\sigma_{d, \text { max }}$, the gain parameter, $\kappa$, and the resonant wavelength, $\lambda$, of the super-resonant asymmetric holey and cake active Ag-based CNRs.

\begin{tabular}{|c|c|c|c|c|c|c|c|c|}
\hline \multirow[t]{2}{*}{$\theta\left[^{\circ}\right]$} & \multicolumn{4}{|c|}{ Holey CNR } & \multicolumn{4}{|c|}{ Cake CNR } \\
\hline & $\sigma_{t}[\mathrm{dBcs}]$ & $\sigma_{d, \max }[\mathrm{dBcs}]$ & $\kappa$ & $\lambda[\mathrm{nm}]$ & $\sigma_{t}[\mathrm{dBcs}]$ & $\sigma_{d, \max }[\mathrm{dBcs}]$ & $\kappa$ & $\lambda[\mathrm{nm}]$ \\
\hline 0 & 67.9 & 71.3 & -0.187 & 576.6 & 67.9 & 71.3 & -0.187 & 576.6 \\
\hline 22.5 & 67.0 & 70.2 & -0.170 & 518.7 & 59.7 & 63.0 & -0.178 & 514.8 \\
\hline 45 & 59.5 & 62.8 & -0.157 & 500.8 & 61.0 & 64.4 & -0.164 & 496.3 \\
\hline 90 & 53.7 & 57.0 & -0.142 & 472.1 & 48.2 & 51.5 & -0.147 & 468.0 \\
\hline
\end{tabular}

asymmetric holey and cake CNRs, see Figure 1a, c, and d, provide a means to achieve more directional responses.

We devote here attention to Ag-based CNR with $h=5 D_{\mathrm{CNR}}$ with the following cut-out angles $\theta=\left\{22.5^{\circ}\right.$, $\left.45^{\circ}, 90^{\circ}\right\}$.

Figure 4 a shows the total scattering cross section, $\sigma_{t}$, as a function of the cut-out angle, $\theta$, for the examined holey and cake CNRs; the corresponding wavelength, $\lambda$, as well as the gain parameter, $\kappa$, are included in Figure $4 \mathrm{~b}$. For easier reference, Table 3 presents a summary of all relevant values for the examined asymmetric holey and cake active Agbased CNRs. For reference purposes, the results for the symmetric $\operatorname{CNR}\left(\theta=0^{\circ}\right)$ are likewise included in the table. Clearly, by suitable adjustments of the gain parameter, super-resonant asymmetric CNRs can also be identified with large total cross section values. The super-resonant behavior is, however, blue-shifted as the particle asymmetry is increased. The edges of the aperture, as well as the additional corner in the cake configuration, along with the associated singular field behaviors lead to the excitation of higher order modes which generally appear at lower wavelengths. This explains the blue-shift of the superresonances in the asymmetric CNRs. Moreover, the results show that less gain is needed as the asymmetry is increased. As the asymmetry is increased, larger portions of the silver nano-shell are removed, thereby diminishing the plasmonic losses although the open geometry leakage losses increase. As a consequence, less gain is needed with an increasing asymmetry. It is also interesting to observe that the holey CNR requires slightly less gain than the cake CNR simply because the gain region of the latter is somewhat physically smaller.

Table 3 includes also the scattering cross section in the maximum direction, $\sigma_{d, \max }$, as determined by (6). As will be clear from the forthcoming results, $\sigma_{d \text { max }}$ is, in all cases, achieved along the positive $x$-axis. Interestingly enough, its values are approximately $3 \mathrm{~dB}$ higher than the corresponding total scattering cross sections in all of the examined cases.

To shed further light on the behavior of asymmetric CNRs, Figure 5 shows the magnetic field, $20 \log |\boldsymbol{H} / 1[\mathrm{~A} / \mathrm{m}]|$, for the super-resonant holey, $h=5 D_{\mathrm{CNR}}$, Ag-based CNR for $\theta=22.5^{\circ}, \theta=45^{\circ}$, and $\theta=90^{\circ}$. The results are shown in the $x y$-plane (upper row) and the $x z$-plane (lower row). Although still at a significantly high level, particularly inside the gainimpregnated cores and close to the nano-shell surfaces, the asymmetry lowers somewhat the overall field levels relative to the symmetric case, see also Figure $3 \mathrm{~b}$ and e. This is in line with the lowered $\sigma_{t}$ values reported in Table 3 . On the other side, a more asymmetric CNR leads to a more directive near-field response, this being particularly so in the vicinity of the respective particles and predominantly into 


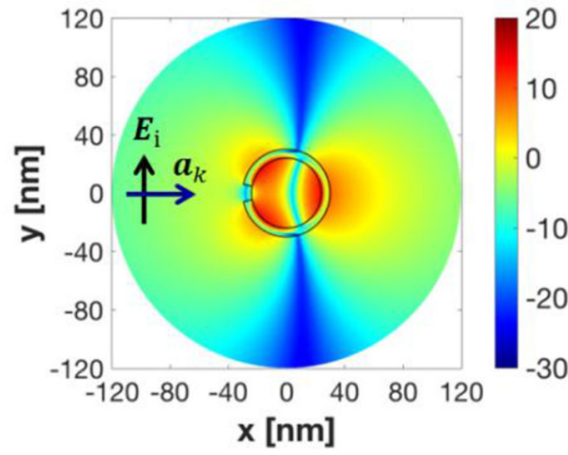

(a)

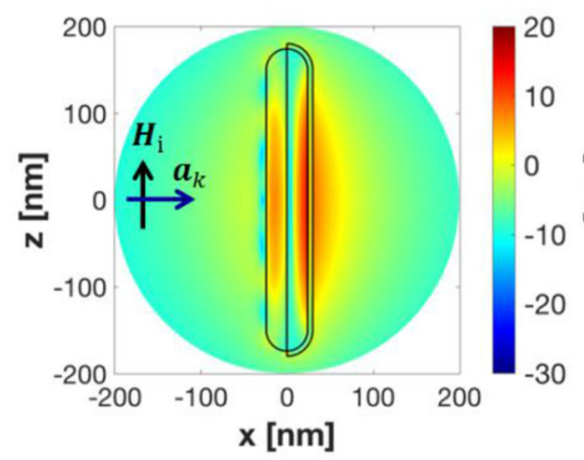

(d)

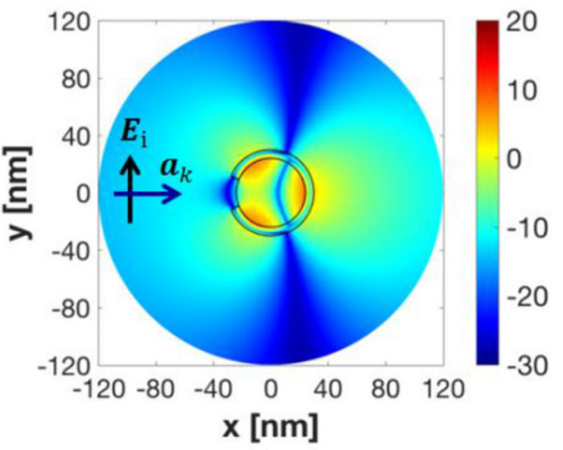

(b)

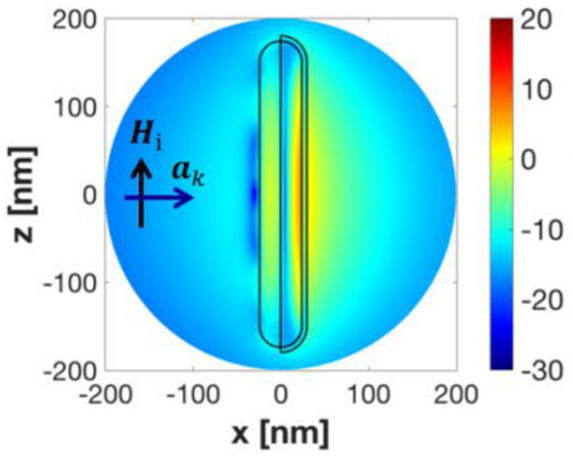

(e)

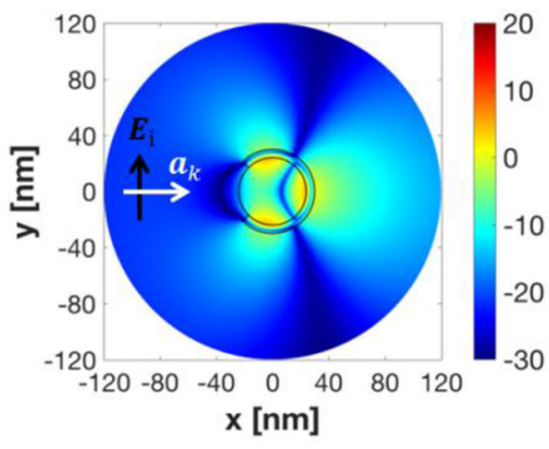

(c)

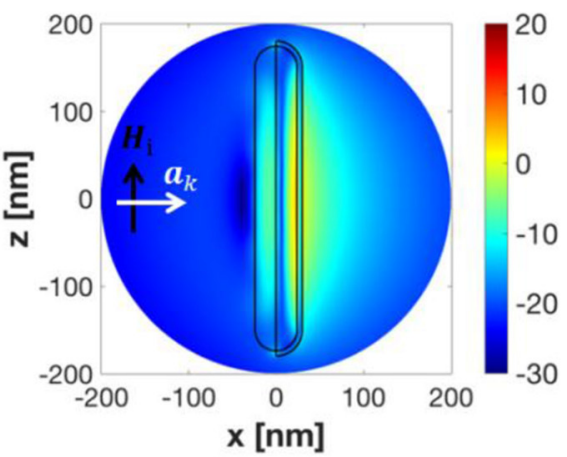

(f)

Fig. 5. The magnetic field, $20 \log |\boldsymbol{H} / 1[\mathrm{~A} / \mathrm{m}]|$, of the super-resonant asymmetric holey Ag-based active CNRs for $\theta=22.5^{\circ}$, (a) and (d); $\theta=45^{\circ}$, (b) and (e); and $\theta=90^{\circ}$, (c) and (f). In all cases, $h=5 D_{\mathrm{CNR}}$. The results are shown in the $x y$-plane (upper row) and the $x z$-plane (lower row). Curves representing the surfaces of the CNRs are included in each figure. The insets depict the direction of the incident fields and wave propagation $\left(\boldsymbol{a}_{k}=\boldsymbol{a}_{x}\right)$.

the half-space opposite to that in which the particle cut-outs have been made and from which the plane wave is being incident. The increased asymmetry obviously leads to a response which is no longer dominated by a dipole mode, as in the symmetric case, but is more due to its mixture with higher order modes, thereby leading to a more directional near-field response. As confirmed by the values of the scattering cross section $\sigma_{d \text { max }}$ reported in Table 3 , the response is not only directional, but also comes with significantly enhanced scattering cross sections in the maximum direction.

The directional nature of the near-fields is next illustrated with specific cuts of the magnetic field as well as the magnitude of the power flow density $\boldsymbol{S}$ defined by (2). The cuts are shown in the $x y$-plane $(z=0)$ as a function of the azimuthal angle $\phi$ when the radial distance $\rho$ to the observation point is set to $40 \mathrm{~nm}$ (Fig. 6a and d), $80 \mathrm{~nm}$ (Fig. $6 \mathrm{~b}$ and e), and $120 \mathrm{~nm}$ (Fig. 6c and f). The upper row shows the magnetic field, while the lower row shows the magnitude of the power flow density.

As can be observed in the figure, these asymmetric CNRs truly lead to rather asymmetric and directional fields (with maximum along the $\phi=0^{\circ}$ direction), this being particularly so for sufficiently near-by observation points. As the observation points recede further away from the structure, the directional features are found to decrease. The higher order modes, which obviously exist in the nearfield region, simply die out and never reach significantly too far away from CNRs. Since the size of these asymmetric CNRs in the $x y$-plane is electrically small, the far-field radiation will attain a dipole mode profile, and thus resembles the one of the symmetric configurations. Similar behavior was observed for spherical all-dielectric [33] and plasmonic [34] particles.

\section{Summary and conclusions}

An extensive numerical analysis of several symmetric and asymmetric active coated nano rod (CNR) antennas was conducted with the aim of identifying the so-called superresonant designs with strongly enhanced but also directive scattering characteristics. The CNRs were of a core-shell type with silica-dioxide nano-core, silver nano-shell, with a constant frequency gain model.

In the symmetric cases, three different lengths of the CNR were examined. Although super-resonant designs were identified, with significantly enhanced scattering cross sections, they were only weakly directive since their responses were due to a strong dipole mode excited in these 


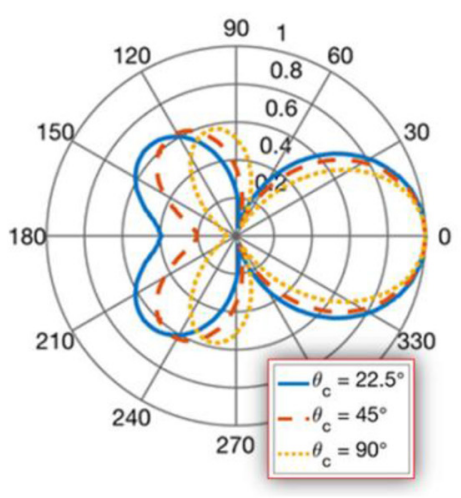

(a)

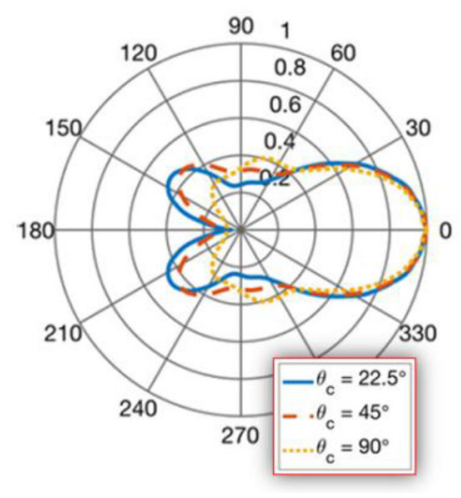

(d)

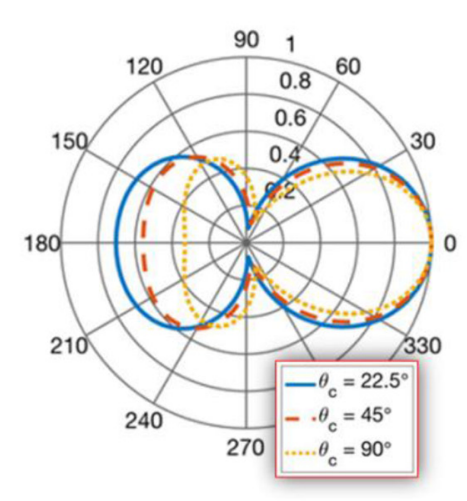

(b)

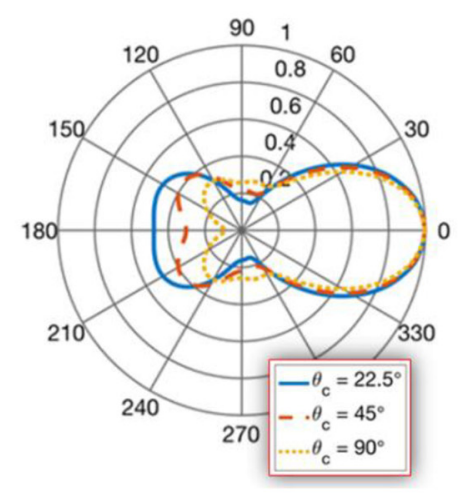

(e)

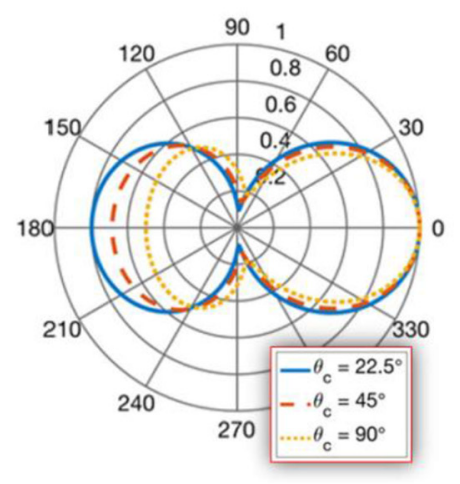

(c)

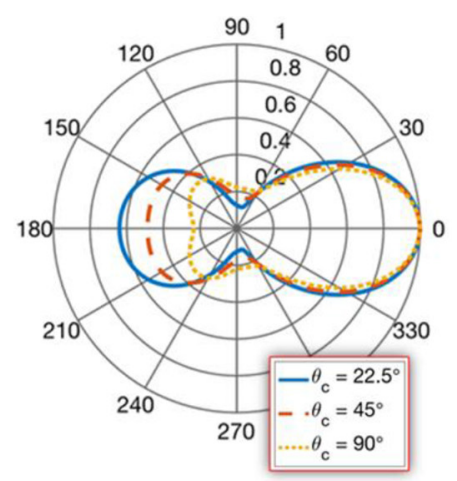

(f)

Fig. 6. The magnetic field, $20 \log |\boldsymbol{H} / 1[\mathrm{~A} / \mathrm{m}]|$ (normalized to its maximum value) (upper row) and the magnitude of the power flow density $\boldsymbol{S}$ (normalized to its maximum value) for a super-resonant, holey Ag-based CNR with $h=5 D_{\mathrm{CNR}}$ and different cut-out angles. The results are shown in the $x y$-plane $(z=0)$ as a function of the observation angle $\phi$ for (a), (d) $\rho=40 \mathrm{~nm},(\mathrm{~b}),(\mathrm{e}) \rho=80 \mathrm{~nm}$, and (c), (f) $\rho=120 \mathrm{~nm}$.

CNRs. The asymmetric CNRs were obtained through three different perforations of the nano-shell and then also of the nano-core. This created edges of the aperture as well as corners in the structure which were necessary to create access to higher order modes. Consequently, the resulting near-fields were found to be very directive in these asymmetric CNRs, this being more pronounced the larger the asymmetry is. However, the higher order modes introduced in this manner gradually die out as the distance to the observation point increases, thereby lowering the directional features. Ultimately, for far-field observation distances, the directive near-field beams will transition into a more typical dipole mode pattern.

The examined CNRs bridge the gap between the two often considered canonical problems, namely, the 2D circular cylindrical and 3D spherical active CNPs. The gradual transition between these geometries via the studied CNRs provides systematic and fundamental insight behind their performance characteristics alongside the required gain values and resonant wavelengths. We found that shorter symmetric CNRs require more gain than the longer ones, and that their resonant wavelengths get blue-shifted as their length decreases. For asymmetric CNRs, the gain is diminished, and the response got blue-shifted as the asymmetry increased. The lower gain levels in the more asymmetric cases are simply due to lower plasmonic losses as they imply larger parts of the silver nano-shell removed. These results are of importance for a successful implementation of such particles with realistic gain media. Apart from provided fundamental insight, the examined CNRs constitute a new class of nano antennas with wavelength tunability and polarization sensitivity, but also with highly enhanced and directional near-field response characteristics. As such they may become interesting candidates for a variety of nano-photonic applications, e.g., optical wireless systems, nano-sensors and single photonic sources. We also note that the observed directional behavior in the proposed CNRs may also be of interest in the design of photonic nano-jets for enhanced near-field directional control $[34,42,43]$.

\section{References}

1. I. Choi, Y. Choi, IEEE J. Sel. Top. Quantum Electron. 18, $1110(2012)$

2. M.T. Hill, J. Opt. Soc. Am. B 27, 36 (2010)

3. Y. Ra'di, V.S. Asadchy, S.U. Kosulnikov, M.M. Omelyanovih, D. Morits, A.V. Osipov, C.R. Simovski, S.A. Tretyakov, ACS Photon. 2, 653 (2015) 
4. A. Monti, A. Toscano, F. Bilotti, Opt. Lett. 41, 3383 (2016)

5. H.A. Atwater, A. Polman, Nat. Mater. 9, 205 (2013)

6. A. Alu, N. Engheta, Nano Lett. 13, 1086 (2013)

7. A. Monti, A. Alu, A. Toscano, F. Bilotti, J. Appl. Phys. 117, $123103(2015)$

8. Z. Su, J. Yin, K. Song, Q. Lei, X. Zhao, Opt. Exp. 24, 6021 (2016)

9. A. Alú, N. Engheta, Phys. Rev. Lett. 104, 213902 (2010)

10. S. Kawata, Y. Inouye, P. Verma, Nat. Photon. 3, 388 (2009)

11. C. Wu, A.B. Khanikaev, R. Adato, N. Arju, A.A. Yanik, H. Altug, G. Shvets, Nat. Mater. 11, 69 (2012)

12. D. Sikdar, I.D. Rukhlenko, W. Cheng, M. Premaratne, Nanoscale Res. Lett. 8, 142 (2013)

13. N.J. Halas, Nano Lett. 10, 3816 (2010)

14. L. Novotny, N. van Hulst, Nat. Photon. 5, 83 (2011)

15. L. Novotny, B. Hecht, Principles of Nano-optics (Cambridge University Press, Cambridge, 2012)

16. M. Agio, A. Alù, Optical antennas (Cambridge University Press, New York, 2013)

17. S.J. Oldenburg, G.D. Hale, J.B. Jackson, N.J. Halas, Appl. Phys. Let. 75, 1063 (1999)

18. S. Kühn, U. Håkanson, L. Rogobete, V. Sandoghdar, Phys. Rev. Lett. 97, 017402 (2006)

19. M. Agio, Nanoscale 4, 692 (2012)

20. A. Alú, N. Engheta, IEEE Trans. Antennas Propag. 61, 1508 (2013)

21. A.B. Evlyukhin, S.M. Novikov, U. Zywietz, R.L. Eriksen, C. Reinhardt, S.I. Bozhevolnyi, B.N. Chichkov, Nano Lett. 12, 3749 (2012)

22. A.E. Krasnok, A.E. Miroshnichenko, P.A. Belov, Y.S. Kivshar, Opt. Exp. 20, 20599 (2012)

23. S. Maier, Plasmonics: fundamentals and applications (Springer, Berlin, 2007)

24. J.A. Gordon, R.W. Ziolkowski, Opt. Exp. 15, 2622 (2007)
25. M. Noginov, G. Zhu, A.M. Belgrave, R. Bakker, V.M. Shalaev, E.E. Narimanov, S. Stout, E. Herz, T. Suteewong, U. Wiesner, Nature 460, 1110 (2009)

26. S. Arslanagić, R.W. Ziolkowski, IEEE Ant. Propag. Mag. 59, $14(2017)$

27. Y.H. Fu, A.E. Krasnok, A.E. Miroshnichenko, Y.F. Yu, B. Luk'yanchuk, Nat. Commun. 4, 1527 (2013)

28. I. Liberal, I. Ederra, R. Gonzalo, R.W. Ziolkowski, Phys. Rev. Appl. 1, 044002 (2014)

29. A. Alù, E. Engheta, IEEE Trans. Antennas Propag. 55, 3027 (2007)

30. Z. Ruan, S. Fan, Appl. Phys. Lett. 98, 04101 (2011)

31. M.W. Knight, N.J. Halas, New J. Phys. 10, 105006 (2008)

32. R.Ø. Thorsen, S. Arslanagić, Photonics 2, 773 (2015)

33. A.E. Krasnok, C.R. Simovski, P.A. Belov, Y.S. Kivshar, Nanoscale 6, 7354 (2014)

34. S.D. Campbell, R.W. Ziolkowski, IEEE J. Sel. Top. Quant. Electron. 21, 4800112 (2014)

35. R. Ziolkowski, Phys. Rev. X 7, 031017 (2017)

36. S. Arslanagić, R.W. Ziolkowski, Phys. Rev. Lett. 120, 237401 (2018)

37. S.Y. Liu, J. Li, F. Zhou, L. Gan, Z.-Y. Li, Opt. Lett. 36, 1296 (2011)

38. J. Geng, R.W. Ziolkowski, R. Jin, X. Liang, IEEE Photon. J. 3, 1093 (2011)

39. J. Geng, R.W. Ziolkowski, R. Jin, X. Liang, J. Electromag. Waves Appl. 27, 1392 (2013)

40. S.D. Campbell, R.W. Zilkowski, Adv. Optoelectron. 2012, 368786 (2012)

41. COMSOL Multiphysics ${ }^{\circledR}$, https://www.comsol.com/

42. A. Heifetz, S.-C. Kong, A.V. Sahakian, A. Tavlove, J. Comput. Theor. Nanosci. 6, 1979 (2009)

43. Y. Huang, Z. Zhen, Y. Shen, C. Min, G. Veronis, Opt. Exp. 27, 1310 (2019)

Cite this article as: Samel Arslanagić, Rasmus E. Jacobsen, Active coated nano rod antennas for enhanced and directive scattering phenomena, EPJ Appl. Metamat. 6, 19 (2019) 